\title{
Gender difference in the association between brachial-ankle pulse wave velocity and cardiovascular risk scores
}

Tae-Min Rhee ${ }^{1,2, \star}$, Hack-Lyoung Kim ${ }^{1,2, \star}$, Sohee Oh${ }^{3}$, Woo-Hyun Lim ${ }^{1,2}$, Jae-Bin Seo ${ }^{1,2}$, Woo-Young Chung ${ }^{1,2}$, Sang-Hyun $\mathrm{Kim}^{1,2}$, Myung-A Kim ${ }^{1,2}$, and Joo-Hee $\mathrm{Zo}^{1,2}$

\begin{abstract}
${ }^{1}$ Department of Internal Medicine, Seoul Metropolitan Government Seoul National University Boramae Medical Center, Seoul; ${ }^{2}$ Department of Internal Medicine, Seoul National University College of Medicine, Seoul; ' ${ }^{3}$ Department of Biostatistics, Seoul Metropolitan Government Seoul National University Boramae Medical Center, Seoul, Korea
\end{abstract}

Received: October 23, 2017

Revised : November 26, 2017

Accepted: December 4, 2017

\section{Correspondence to}

Joo-Hee Zo, M.D.

Division of Cardiology, Department of Internal Medicine, Seoul Metropolitan Government Seoul National University Boramae Medical Center, 20 Boramae-ro 5-gil, Dongjak-gu, Seoul 07061, Korea

Tel: +82-2-870-2114

Fax: +82-2-870-3866

E-mail:jooheezo@hanmail.net

*These authors contributed equally to this work.
Background/Aims: Although brachial-ankle pulse wave velocity (baPWV) has been validated as a novel method to predict the cardiovascular risk in general population, the relevance of baPWV to the traditional risk scores has not been clearly revealed. This study investigated the relationship between baPWV and four different cardiovascular risk-predicting scores in men and women.

Methods: A total of 539 subjects $(58.1 \pm 12.2$ years, $50.1 \%$ men) without cardiovascular disease (CVD) who underwent health examinations including baPWV measurement were retrospectively analyzed. Four cardiovascular risk scores (Framingham risk score [FRS; 1998], Adult Treatment Panel [ATP] III revised FRS [2002], generalized FRS [2008], and American College of Cardiology/American Heart Association [ACC/AHA] CVD risk [2013]) were calculated in each subject.

Results: In a total population, baPWV was moderately correlated with four cardiovascular risk scores $(r=0.577$ for FRS; $r=0.594$ for ATP III revised FRS; $r=0.589$ for generalized FRS; $r=0.571$ for ACC/AHA CVD risk; $p<0.001$ for each). These correlations were stronger in women than in men ( $r=0.649$ vs. 0.451 for FRS; $r=$ 0.719 vs. 0.411 for ATP III revised FRS; $r=0.735$ vs. 0.540 for generalized FRS; $r=$ 0.699 vs. 0.552 for ACC/AHA CVD risk; $p$ for gender difference $\leq 0.005$ for each).

Conclusions: In middle-aged and elderly Koreans without CVD, baPWV was identified as having a moderately positive correlation with four different risk scores. The correlation was stronger in women than in men, implying the better performance of baPWV in women for predicting cardiovascular risk of healthy population.

Keywords: Cardiovascular diseases; Risk assessment; Pulse wave analysis; Sex characteristics

\section{INTRODUCTION}

Arterial stiffness, reflecting structural changes of the arterial wall, has been widely used as a marker of vascular damage and arteriosclerosis [1]. Data on arterial stiffness is clinically important because arterial stiffness is an independent risk factor of cardiovascular (CV) morbidity and mortality in various medical conditions as well as in the general population [2-5]. Arterial stiffness can be assessed noninvasively by measuring pulse wave velocity (PWV), which is a simple and reliable method [6]. The most frequently studied index among several PWV 
measures is carotid-femoral pulse wave velocity (cfPWV) [7]. However, application of cfPWV is limited, because of its technical difficulty and inconvenience during the measurement. As a more recently developed method, the brachial-ankle pulse wave velocity (baPWV) has been emerged as an easier, less time-consuming, and less stressful screening tool compared to cfPWV [8]. Moreover, baPWV shows a good correlation with cfPWV [9], and clinical usefulness of baPWV has been validated in many clinical studies [10-13] and meta-analysis [14]. Therefore, baPWV is useful especially in mass screening, and its application has rapidly spread throughout East Asian countries, including Japan and South Korea.

Cardiovascular disease (CVD) constitutes a major public health problem showing high prevalence and mortality. Investigators have developed various multivariable risk-predicting algorithms to predict the future CV events and classify the CV high-risk population. In 1998, the Framingham Heart Study Group suggested an algorithm able to estimate a 10-year risk of coronary heart disease (CHD) [15]. Following the Framingham risk score (FRS), several multivariate risk-predicting system with slightly different primary outcomes have been introduced. In 2002, the National Cholesterol Education Program Expert Panel published the Adult Treatment Panel III (ATP III) for the management of high blood cholesterol, presenting the revised FRS targeting a 10-year risk of hard CHD, which was composed of only myocardial infarction and CHD death [16]. Later in 2008, the Framingham Heart Study Group generalized its outcome to the 10-year risk of atherosclerotic cardiovascular disease (ASCVD), covering CHD, stroke, peripheral arterial disease, and congestive heart failure [17]. In 2013, the American College of Cardiology/American Heart Association (ACC/AHA) guideline on the assessment of CV risk suggested an equation to approximate a 10-year risk for the first hard ASCVD, including nonfatal myocardial infarction/CHD death and nonfatal/fatal stroke [18].

However, along with the development of new diagnostic technologies rescuing human being from CV risks, there have been critics against traditional risk scores, presenting the inaccuracy of prior multivariable CV risk prediction models [19]. In fact, many CV events occur in individuals who were not identified as high-risk patients by previous CV risk scores in real-world [20]. In this context, newer methods have been introduced to increase the capability of CV risk. As one of those novel tools, PWV has been investigated in many clinical studies, being revealed to have the value in the prediction of future CVD [21,22].

To date, data on direct comparison between PWV and $\mathrm{CV}$ risk scores have been limited [15]. It is necessary to realize the relevance of PWV to a risk score before revealing the prognostic power of PWV. Therefore, the purpose of this study is to investigate the relationship between the baPWV value and aforementioned CV risk-predicting scores.

\section{METHODS}

\section{Study population}

Between September 2012 and July 2013, consecutive subjects who visited Seoul Metropolitan Government Seoul National University Boramae Medical Center (Seoul, Korea) for health examination were retrospectively reviewed. A total of 819 subjects who underwent baPWV measurement as part of CV examinations were initially screened for the study. Subjects with a history of previous CHD or stroke $(\mathrm{n}=132)$, those with unavailable information to calculate the $\mathrm{CV}$ risk ( $\mathrm{n}=$ 108), those with atrial fibrillation $(\mathrm{n}=23)$ and lower ankle brachial index (ABI; < 0.9) ( $\mathrm{n}=17)$ were excluded from the study, leaving 539 subjects to be finally analyzed. All study subjects were medically stable at the time of health checkup. The information on medical history, including hypertension, diabetes mellitus, dyslipidemia, and smoking habits, was obtained by a standardized questionnaire. Body mass index (BMI) was calculated as body weight (kg) divided by the square of body height $(\mathrm{m})$. Systolic and diastolic blood pressures (SBP and DBP) were measured at right upper arm by a trained nurse. All subjects underwent laboratory tests by sampling venous blood in the morning after overnight fasting. White blood cell (WBC) count, hemoglobin, concentration and serum levels of fasting glucose, total cholesterol, low density lipoprotein cholesterol (LDL-C), high density lipoprotein cholesterol (HDL-C), triglyceride, creatinine, and C-reactive protein were measured by an automated enzymatic procedure. Estimated glomerular filtration rate was calculated using four-component 


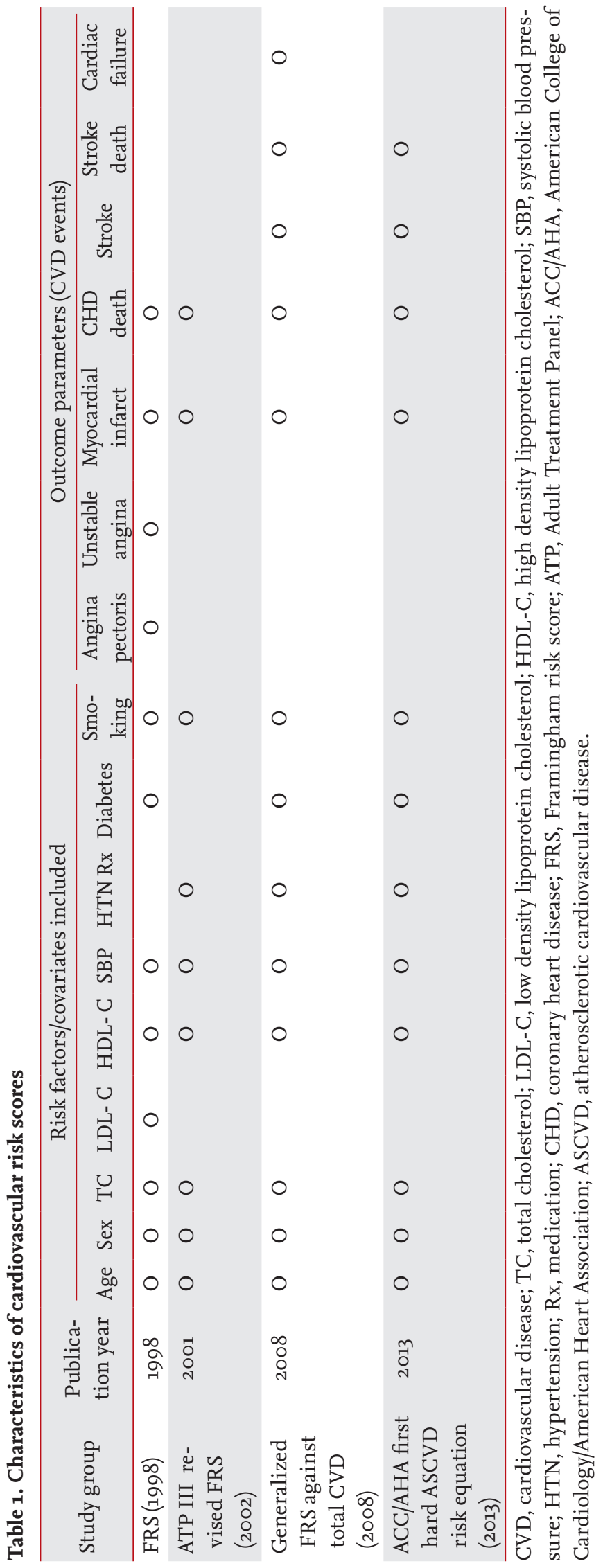

MDRD (Modification of Diet in Renal Disease study) equation incorporating age, race, sex, and serum creatinine level [23].

\section{baPWV measurement}

In this study, baPWV was measured by using the previously described protocol [12]. Regular medications, caffeine ingestion and cigarette smoking were not allowed before the measurement. All measurements were conducted in a quiet room at a constant temperature. Subjects were examined in the supine position after at least 5 minutes of rest, and baPWV was measured by a volume-plethysmographic apparatus (VP-1000, Colin Co. Ltd., Komaki, Japan) according to the manufacturer's instructions. Phonogram and pulse volume waveform were stored with cuffs placed around both the brachia and the ankles. The mean value of the right- and left-sided baPWV was used for the analysis. Blood pressure and heart rate were measured and recorded by the same device. Each of baPWV values measurements was performed by the same experienced investigator who was blinded to the subjects' clinical information. The intraobserver coefficient of variation of the baPWV measurement was about $5 \%$ in our laboratory.

\section{Calculation of $\mathrm{CV}$ risk scores}

Four different scoring systems (FRS [1998], ATP III revised FRS [2002], generalized FRS against total CVD [2008], and ACC/AHA first hard ASCVD risk equation [2013]) were used to calculate the estimates of each subject's CV risk in the next 10 years. Table 1 shows the characteristics of these risk scores, which includes data source, covariates, and outcome parameters. In this study, risk scores were calculated from age, gender, the status of hypertension, diabetes mellitus, smoking, and blood levels of total or LDL-C and HDL-C. Hypertension was defined as the use of anti-hypertensive medications or SBP $\geq 140$ $\mathrm{mmHg}$ and/or DBP $\geq 90 \mathrm{mmHg}$. Diabetes mellitus was defined as the use of oral hypoglycemic medications or insulin or serum fasting glucose level $\geq 126$ $\mathrm{mg} / \mathrm{dL}$. Subjects were classified as smokers if they had smoked regularly during previous 12 months. 
Table 2. Clinical characteristics of study population

\begin{tabular}{|c|c|c|c|c|}
\hline Characteristic & Total $(\mathrm{n}=539)$ & $\operatorname{Men}(\mathrm{n}=270)$ & Women $(n=269)$ & $p$ value $^{a}$ \\
\hline Age, yr & $58.1 \pm 12.2$ & $55.9 \pm 12.1$ & $60.3 \pm 12.0$ & $<0.001$ \\
\hline Height, cm & $162 \pm 9$ & $168 \pm 6$ & $155 \pm 6$ & $<0.001$ \\
\hline Weight, kg & $65.5 \pm 12.3$ & $72.6 \pm 11.5$ & $58.5 \pm 8.6$ & $<0.001$ \\
\hline $\mathrm{BMI}, \mathrm{kg} / \mathrm{m}^{2}$ & $24 \cdot 7 \pm 3 \cdot 4$ & $25 \cdot 3 \pm 3.1$ & $24.1 \pm 3.5$ & $<0.001$ \\
\hline $\mathrm{SBP}, \mathrm{mmHg}$ & $126 \pm 14$ & $126 \pm 13$ & $127 \pm 16$ & 0.184 \\
\hline $\mathrm{DBP}, \mathrm{mmHg}$ & $76.3 \pm 9.4$ & $77.5 \pm 9.1$ & $75.2 \pm 9.5$ & 0.005 \\
\hline \multicolumn{5}{|l|}{ Traditional risk factors } \\
\hline Hypertension & $284(52.7)$ & $140(51.9)$ & $144(53 \cdot 5)$ & 0.696 \\
\hline Diabetes mellitus & $92(17.1)$ & $46(17.0)$ & $46(17.1)$ & 0.984 \\
\hline Dyslipidemia & $313(58.1)$ & $155(57 \cdot 4)$ & $158(58.7)$ & 0.755 \\
\hline Smoking & $136(25.2)$ & $133(49 \cdot 3)$ & $3(1.1)$ & $<0.001$ \\
\hline \multicolumn{5}{|l|}{ Laboratory findings } \\
\hline $\mathrm{WBC}, / \mu \mathrm{L}$ & $6,485 \pm 1,819$ & $6,793 \pm 1,897$ & $6,171 \pm 1,681$ & $<0.001$ \\
\hline Hemoglobin, g/dL & $14.0 \pm 1.6$ & $14.9 \pm 1.4$ & $13.0 \pm 1.2$ & $<0.001$ \\
\hline Fasting glucose, mg/dL & $105 \cdot 5 \pm 23 \cdot 5$ & $105.7 \pm 20.8$ & $105.2 \pm 25.9$ & 0.796 \\
\hline Total cholesterol, mg/dL & $185 \pm 36$ & $183 \pm 38$ & $187 \pm 33$ & 0.275 \\
\hline HDL-C, mg/dL & $49.2 \pm 11.1$ & $45 \cdot 6 \pm 9.9$ & $52.8 \pm 11.1$ & $<0.001$ \\
\hline LDL-C, mg/dL & $117 \pm 34$ & $117 \pm 35$ & $116 \pm 32$ & 0.801 \\
\hline Triglyceride, mg/dL & $127 \pm 69$ & $139 \pm 75$ & $115 \pm 60$ & $<0.001$ \\
\hline hs-CRP, mg/dL & $0.22 \pm 0.48$ & $0.25 \pm 0.53$ & $0.18 \pm 0.40$ & 0.328 \\
\hline $\mathrm{eGFR}, \mathrm{mL} / \mathrm{min} / 1.73 \mathrm{~m}^{2}$ & $87.6 \pm 19.5$ & $87.6 \pm 17.8$ & $87.6 \pm 21.1$ & 0.998 \\
\hline baPWV, cm/sec & $1,511 \pm 253$ & $1,480 \pm 224$ & $1,542 \pm 275$ & 0.005 \\
\hline Framingham risk score (1998) & $6.0(4.0-8.0)$ & $6.0(3.75-7.0)$ & $7.0(4.0-10.0)$ & $<0.001$ \\
\hline ATP III revised FRS (2002) & $13.0(10.0-15.0)$ & $12.0(10.0-14.0)$ & $14.0(11.0-17.0)$ & $<0.001$ \\
\hline Generalized FRS against total CVD (2008) & $13.0(9.0-16.0)$ & $14.0(10.0-17.0)$ & $12.0\left(7.5^{-15} \cdot 0\right)$ & $<0.001$ \\
\hline $\begin{array}{l}\text { ACC/AHA first hard ASCVD risk } \\
\text { equation (2013) }\end{array}$ & $8.1(3.2-16.2)$ & $10.4(5.4-18.7)$ & $5 \cdot 7(1.8-13.1)$ & $<0.001$ \\
\hline
\end{tabular}

Values are presented as mean $\pm \mathrm{SD}$, number (\%), or median (interquartile range).

BMI, body mass index; SBP, systolic blood pressure; DBP, diastolic blood pressure; WBC, white blood cell; HDL-C, high density lipoprotein cholesterol; LDL-C, low density lipoprotein cholesterol; hs-CRP, high-sensitivity C-reactive protein; eGFR, estimated glomerular filtration rate; baPWV, brachial-ankle pulse wave velocity; ATP, Adult Treatment Panel; FRS, Framingham risk score; CVD, cardiovascular disease; ACC/AHA, American College of Cardiology/American Heart Association; ASCVD, atherosclerotic cardiovascular disease.

${ }^{a} p$ value was obtained from the comparison between men and women.

\section{Statistical analysis}

Continuous variables were expressed as mean \pm standard deviation (SD) or median with interquartile range, and categorical variables as number with percentages. Comparison of means and proportions between genders was performed using Student $t$ test or Mann-Whitney test and the chi-square test, as the type of variables. As CV risk scores were based on ranks without the assumption of normal distribution, Spearman's rank correlation analyses were used to investigate the power of linear correlation between risk scores and baPWV. The correlation was displayed by scatter plots. Gender difference of the correlation coefficients was analyzed by Fischer r-to-Z-transformation method. Linear correlation anal- 
Table 3. Correlations between brachial-ankle pulse wave velocity and cardiovascular risk scores

\begin{tabular}{|c|c|c|c|c|}
\hline \multirow{2}{*}{ Cardiovascular risk scores } & \multirow{2}{*}{ Total $(\mathrm{n}=539)$} & \multicolumn{3}{|c|}{ Simple linear correlations } \\
\hline & & $\operatorname{Men}(n=270)$ & Women $(n=269)$ & $p$ value $^{\mathrm{a}}$ \\
\hline FRS (1998) & $0.577^{\mathrm{b}}$ & $0.451^{b}$ & $0.649^{b}$ & $<0.001$ \\
\hline ATP III revised FRS (2002) & $0.594^{\mathrm{b}}$ & $0.411^{b}$ & $0.719^{\mathrm{b}}$ & $<0.001$ \\
\hline Generalized FRS against total CVD (2008) & $0.589^{b}$ & $0.540^{b}$ & $0.735^{\mathrm{b}}$ & $<0.001$ \\
\hline ACC/AHA first hard ASCVD risk equation (2013) & $0.571^{b}$ & $0.552^{b}$ & $0.699^{b}$ & 0.005 \\
\hline
\end{tabular}

FRS, Framingham risk score; ATP, Adult Treatment Panel; CVD, cardiovascular disease; ACC/AHA, American college of cardiology/American heart association; ASCVD, atherosclerotic cardiovascular disease.

a $p$ value for difference was obtained from the comparison of correlations between men and women by using the Fisher's Z-transformation method.

${ }^{\mathrm{b}}$ Correlation was significant at the 0.001 level (2-tailed).

Table 4. Correlations between brachial-ankle pulse wave velocity and conventional cardiovascular risk factors

\begin{tabular}{|c|c|c|c|c|}
\hline \multirow{2}{*}{ Variable } & \multicolumn{2}{|c|}{$\operatorname{Men}(\mathrm{n}=270)$} & \multicolumn{2}{|c|}{ Women $(\mathrm{n}=269)$} \\
\hline & $r$ & $p$ value & $r$ & $p$ value \\
\hline Age, yr & 0.457 & $<0.001$ & 0.607 & $<0.001$ \\
\hline $\mathrm{BMI}, \mathrm{kg} / \mathrm{m}^{2}$ & -0.051 & 0.422 & 0.194 & 0.002 \\
\hline $\mathrm{SBP}, \mathrm{mmHg}$ & 0.599 & $<0.001$ & 0.616 & $<0.001$ \\
\hline $\mathrm{DBP}, \mathrm{mmHg}$ & 0.429 & $<0.001$ & 0.361 & $<0.001$ \\
\hline Fasting glucose, mg/dL & 0.153 & 0.012 & 0.311 & $<0.001$ \\
\hline Total cholesterol, mg/dL & -0.056 & 0.358 & -0.094 & 0.124 \\
\hline HDL-C, mg/dL & -0.014 & 0.823 & -0.200 & 0.001 \\
\hline LDL-C, mg/dL & -0.053 & 0.383 & -0.079 & 0.196 \\
\hline Triglyceride, mg/dL & -0.063 & 0.306 & 0.212 & $<0.001$ \\
\hline
\end{tabular}

BMI, body mass index; SBP, systolic blood pressure; DBP, diastolic blood pressure; HDL-C, high density lipoprotein cholesterol; LDL-C, low density lipoprotein cholesterol.

yses (Pearson or Spearman, along with the distributive nature of variables) between baPWV and conventional $\mathrm{CV}$ risk factors were then followed. A p value $<0.05$ was considered statistically significant. For statistical analyses, we used a SPSS version 19 (IBM Co., Armonk, NY, USA) and R version 3.1.2 (http://www.r-project.org).

\section{Ethics statement}

An approval for the study protocol was obtained from the Institutional Review Board (IRB) of Seoul Metropolitan Government Seoul National University Boramae Medical Center (Seoul, Korea) (IRB No. 16-2013-23). Informed consent was waived by IRB due to the routine nature of the information collected and retrospective study design.

\section{RESULTS}

Table 2 shows clinical characteristics of the study population by gender. The mean age was $58.1 \pm 12.2$ years, and 269 (49.9\%) were women. The proportions of the study subjects with hypertension, diabetes mellitus, and dyslipidemia were $52.7 \%, 17.1 \%$, and $58.1 \%$, respectively. About a quarter of them were smokers. Women were older $(60.3 \pm 12.0$ years vs. $55.9 \pm 12.1$ years, $p<0.001)$, and had lower BMI than men. DBP was lower in women than in men. The incidence of traditional risk factors, including hypertension, diabetes mellitus, and dyslipidemia were not different in both genders, except a lower proportion of smokers in women than men. In laboratory findings, women showed lower WBC count, hemoglobin concentration and triglyceride level, and higher 
Men
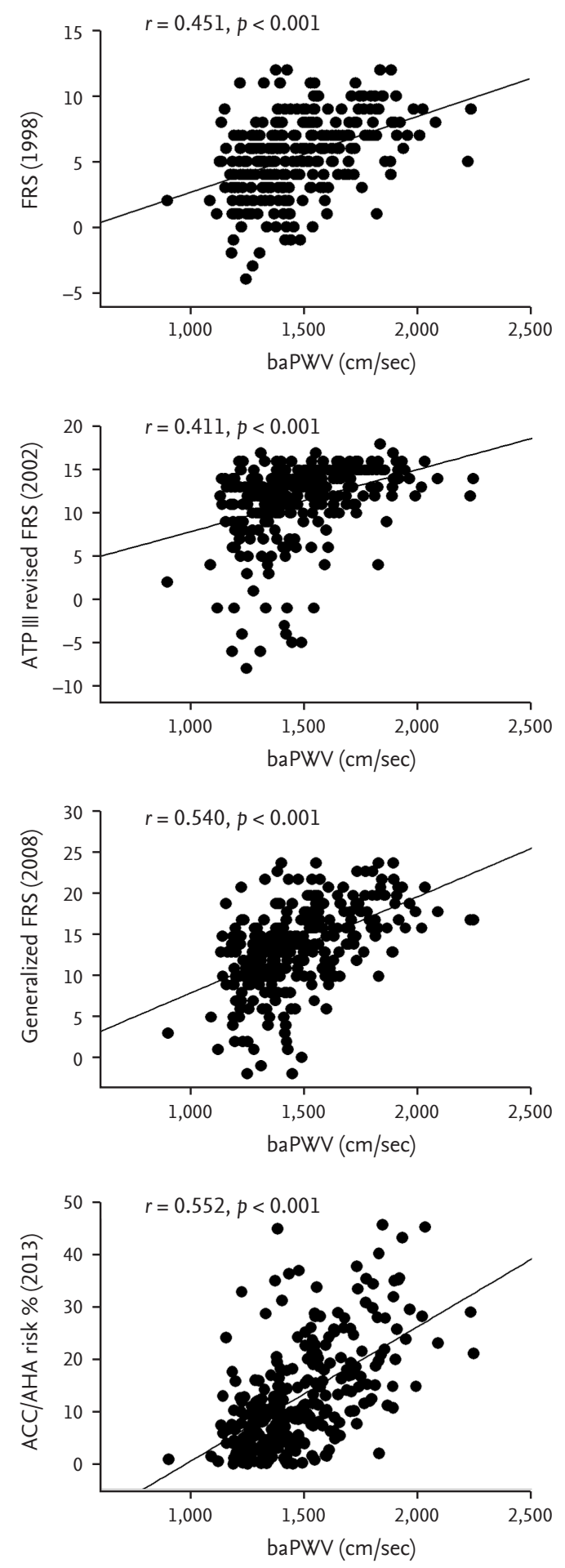

Women
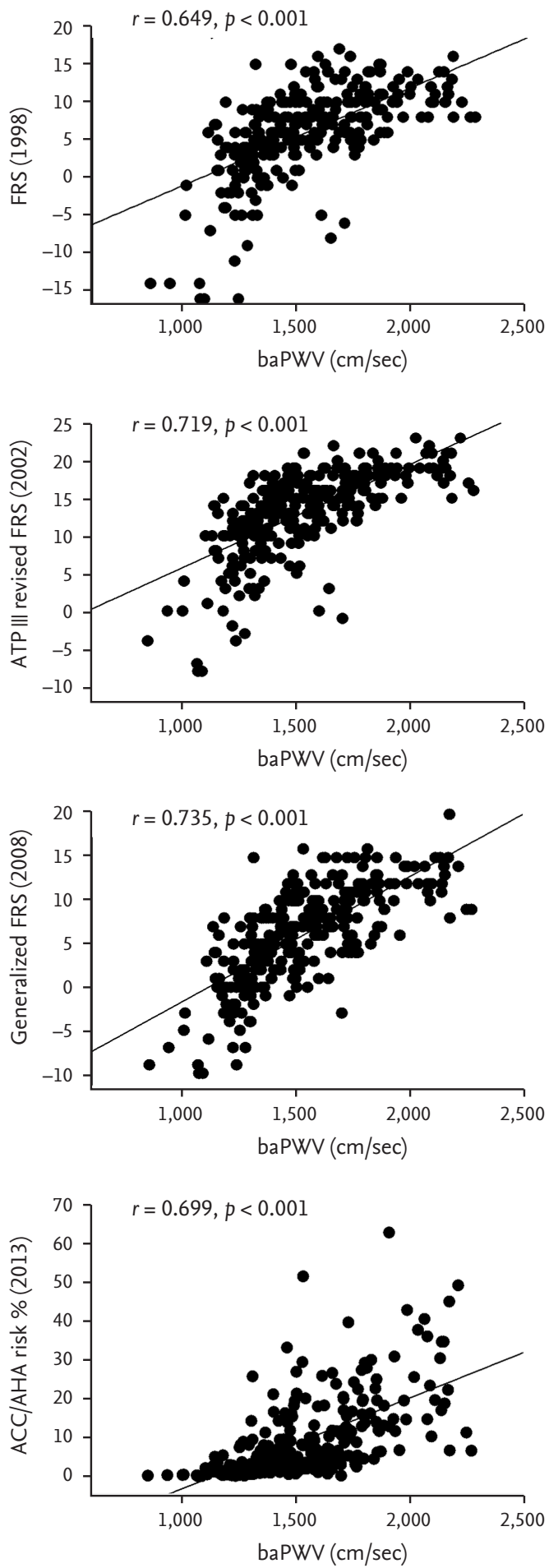

Figure 1. Scatter plots of linear correlations between brachial-ankle pulse wave velocity (baPWV) and cardiovascular risk scores. The scatter plots briefly demonstrate the significant between-gender difference of the degree of correlations ( $r$, correlation coefficient). FRS, Framingham risk score; ATP, adult treatment panel; ACC/AHA, American College of Cardiology/American Heart Association. 
HDL-C level than men. The mean value of baPWV was higher in women than in men $(1,542 \pm 275 \mathrm{~cm} / \mathrm{sec}$ vs. $1,480 \pm 224 \mathrm{~cm} / \mathrm{sec}, p=0.005)$. Ten-year CV risk scores from FRS (1998) and ATP III revised FRS (2002) were also higher in women than in men. In contrast, those from generalized FRS against total CVD (2008) and ACC/AHA first hard ASCVD risk equation (2013) were higher in men than in women.

The baPWV value showed moderate positive correlations with all four CV risk scores (correlation coefficient $[r]=0.577$ for FRS [1998]; $r=0.594$ for ATP III revised FRS [2002]; $r=0.589$ for generalized FRS against total CVD [2008]; and $r=0.571$ for ACC/AHA first hard ASCVD risk equation [2013]; with $p$ values $<0.001$ for each). Stronger correlations were observed in women than in men ( $p$ for gender difference $\leq 0.005$ for each) (Table 3 ). Simple linear correlations between baPWV and CV risk scores are displayed by scatter plots in Fig. 1.

Table 4 shows the correlations between baPWV and conventional CV risk factors, most of which are included in CV risk scores. Age, fasting glucose level, SBP, and DBP were significantly correlated with baPWV in both men and women, while BMI, HDL-C, and triglyceride levels were significantly correlated only in women.

\section{DISCUSSION}

The results of this study demonstrated that baPWV is moderately correlated with four different CV risk scores, and that the correlation powers were significantly stronger in women than in men for middle-aged and elderly Korean population without documented CVD. To the best of our knowledge, this is the first report demonstrating direct relationship between baPWV and various $\mathrm{CV}$ risk scores in the view of gender difference. Our results imply the possible role of baPWV in the prediction of CV risk in healthy adults, especially for women.

Several studies have shown positive associations between arterial stiffness and CV risk scores. However, most of those studies used only FRS (1998), with primary outcome limited to CHD $[8,24,25]$. Recent prospective studies have found that PWV is helpful in predicting the risk of ASCVD, including stroke and congestive heart failure as well as CHD [14,21,22,26,27]. There have been several CV risk-predicting algo- rithms of which primary outcomes were different from that of the original FRS. However, studies on the correlation between arterial stiffness and revised or newly introduced risk scores have been scarce until now. In our study, moderate correlations of baPWV with various risk scores were found, suggesting that baPWV can be a useful predictor for assessing the risk of not only CHD, but also generalized ASCVD. Correlation power between baPWV and FRS in our study was similar to that of previous studies $[8,24]$.

We observed significant gender differences in the correlation between baPWV and CV risk scores. The correlation coefficients were stronger in women than in men, suggesting that the CV risk predictability of baPWV could be better in women than in men. Consistent with our findings, there have been several previous studies indicating the gender difference of $\mathrm{CV}$ risk-predictive value of screening tools. A meta-analysis has shown that addition of ABI to FRS results in a higher substantial reclassification rate in women than in men [28]. Nambi et al. [29] have found that the additive CV risk predictive value of intima-media thickness and the presence of plaque of carotid artery are higher in women. Higashi et al. [30] have also found that left ventricular diastolic function has a stronger association with baPWV in healthy women than in healthy men. There is additional evidence to support that the relationship between PWV and CV parameters is stronger in women than in men. In our previous study, we reported that the effects of metabolic syndrome and its components on baPWV are more pronounced in women than in men [11]. Holewijn et al. [31] have also demonstrated a higher additive prognostic value of cfPWV in women compared to men. Along with these findings, our result highlights the evidence to support more powerful value of baPWV in women than in men. In contrast, a few studies have reported that improvement of CV risk predictability by addition of cfPWV to standard risk factors was independent of gender $[32,33]$. These conflicting findings emphasize the need for further research with a special focus on the gender difference of $\mathrm{CV}$ risk prediction with PWV.

The pathophysiology of the gender difference in CV risk prediction has not yet been clearly understood. Our results showed that age and blood pressure were 
significantly correlated with baPWV in both genders, but BMI, fasting glucose, HDL-C, and triglyceride level showed significant correlations with baPWV only in women, which is a similar result with a study by Fujiwara et al. [34]. This finding implies that, especially in women, various $\mathrm{CV}$ and metabolic risk factors other than blood pressure and age are closely related to baPWV. It is therefore not surprising that correlations between baPWV and risk scores, composite of traditional risk factors, were more pronounced in women. In addition, there was a possibility that several factors such as older age and lower prevalence of cigarette smoking in women, as shown in our study, may contribute to the gender difference. Increased differences in CV risk patterns in women than men with aging and hormonal changes may be another important factor affecting the gender difference [35]. Further studies on precise mechanism responsible for the gender difference are warranted.

To date, the cfPWV value has been considered the gold standard noninvasive method to assess arterial stiffness [7]. However, cfPWV measurement demands operator's skill and causes patient's inconvenience during the examination of the carotid and femoral arteries. Otherwise, baPWV measurement is simple by wrapping the cuffs around brachial and posterior tibial arteries. Regardless of the difference in measuring procedure, baPWV is well correlated with cfPWV [9] and invasive methods [36]. More important, prognostic value of baPWV has been validated in many clinical studies $[10,37]$ and meta-analyses [14]. Therefore, baPWV has been emerged as a more effective way to assess arterial stiffness in large-scale populations [38]. Our study showed that the correlation between baPWV and CV risk scores was more powerful in women than in men, implying that the gender effect should be considered when we use baPWV to assess CVD risk. Also, it has been suggested that traditional risk factors do not properly reflect patients' risk, notably for women [39]. In this context, further longitudinal studies focusing on gender differences are warranted to clarify if baPWV can be used as a more useful predictive tool especially in women.

Besides retrospective design, this study has several limitations. First, the study was cross-sectional, and clinical follow-up data were not available. By these reasons, the association between baPWV and CV risk scores cannot certainly indicate a causal relationship, and the predictive value of baPWV could not be directly assessed. Second, as the data regarding medications are missing, the effect of drugs such as anti-hypertensive and lipid-lowering agents on baPWV was ignored in our study. Third, as the target population of the CV risk scores used in our study was mainly non-Hispanic Whites and African-Americans, there would be some discrepancy applying the algorithms to Koreans. Finally, as our data was from middle-aged and elderly Korean, generalization to other population is difficult.

In conclusion, in middle-aged and elderly Korean adults without a history of pre-existing CVD, baPWV had a moderately positive correlation with four different CV risk scores. However, the correlations were more powerful in women than in men. Thus, baPWV could perform better in predicting CV risk of healthy population, especially for women. Longitudinal studies with a larger sample size are needed to confirm our findings.

\section{KEY MESSAGE}

1. Although brachial-ankle pulse wave velocity (baPWV) is a novel method to evaluate the cardiovascular risk, the relationship between baPWV and traditional cardiovascular risk factors has not been clearly revealed.

2. baPWV was moderately correlated with four different cardiovascular risk scores, and the correlation powers were significantly stronger in women than in men for Korean population without documented cardiovascular disease.

3. baPWV could be a handy and useful tool to predict the future cardiovascular risk of healthy population, especially for women.

\section{Conflict of interest}

No potential conflict of interest relevant to this article was reported.

\section{REFERENCES}

1. Lee HY, Oh BH. Aging and arterial stiffness. Circ J 
2010;74:2257-2262.

2. Mitchell GF, Hwang SJ, Vasan RS, et al. Arterial stiffness and cardiovascular events: the Framingham Heart Study. Circulation 2010;121:505-511.

3. Chen BW, Wang ZG, Liu Q, Wang X, Dang A. Combination of pulse wave velocity with clinical factors as a promising tool to predict major adverse cardiac events after percutaneous coronary intervention. J Cardiol 2015;65:318-323.

4. Kimura K, Tomiyama H, Matsumoto C, et al. Correlations of arterial stiffness/central hemodynamics with serum cardiac troponin $\mathrm{T}$ and natriuretic peptide levels in a middle-aged male worksite cohort. J Cardiol 2015;66:135142.

5. Sugamata W, Nakamura T, Uematsu M, et al. Combined assessment of flow-mediated dilation of the brachial artery and brachial-ankle pulse wave velocity improves the prediction of future coronary events in patients with chronic coronary artery disease. J Cardiol 2014;64:179-184.

6. Asmar R, Benetos A, Topouchian J, et al. Assessment of arterial distensibility by automatic pulse wave velocity measurement. Validation and clinical application studies. Hypertension 1995;26:485-490.

7. Laurent S, Cockcroft J, Van Bortel L, et al. Expert consensus document on arterial stiffness: methodological issues and clinical applications. Eur Heart J 2006;27:2588-2605.

8. Mitani S, Fujita M, Shigeta M, et al. Determinants of brachial-ankle pulse wave velocity in a Japanese population: a cohort study. Blood Press 2012;21:338-344.

9. Tanaka H, Munakata M, Kawano Y, et al. Comparison between carotid-femoral and brachial-ankle pulse wave velocity as measures of arterial stiffness. J Hypertens 2009;27:2022-2027.

10. Sheng CS, Li Y, Li LH, et al. Brachial-ankle pulse wave velocity as a predictor of mortality in elderly Chinese. Hypertension 2014;64:1124-1130.

11. Kim HL, Lee JM, Seo JB, et al. The effects of metabolic syndrome and its components on arterial stiffness in relation to gender. J Cardiol 2015;65:243-249.

12. Kim HL, Im MS, Seo JB, et al. The association between arterial stiffness and left ventricular filling pressure in an apparently healthy Korean population. Cardiovasc Ultrasound 2013;11:2.

13. Park KT, Kim HL, Oh S, et al. Association between reduced arterial stiffness and preserved diastolic function of the left ventricle in middle-aged and elderly patients. J Clin Hypertens (Greenwich) 2017;19:620-626.
14. Vlachopoulos C, Aznaouridis K, Terentes-Printzios D, Ioakeimidis N, Stefanadis C. Prediction of cardiovascular events and all-cause mortality with brachial-ankle elasticity index: a systematic review and meta-analysis. Hypertension 2012;60:556-562.

15. Wilson PW, D'Agostino RB, Levy D, Belanger AM, Silbershatz H, Kannel WB. Prediction of coronary heart disease using risk factor categories. Circulation 1998;97:1837-1847.

16. National Cholesterol Education Program (NCEP) Expert Panel on Detection, Evaluation, and Treatment of High Blood Cholesterol in Adults (Adult Treatment Panel III). Third report of the National Cholesterol Education Program (NCEP) expert panel on detection, evaluation, and treatment of high blood cholesterol in adults (Adult Treatment Panel III) final report. Circulation 2002;106:3143-3421.

17. D'Agostino RB Sr, Vasan RS, Pencina MJ, et al. General cardiovascular risk profile for use in primary care: the Framingham Heart Study. Circulation 2008;117:743-753.

18. Goff DC Jr, Lloyd-Jones DM, Bennett G, et al. 2013 ACC/ AHA guideline on the assessment of cardiovascular risk: a report of the American College of Cardiology/American Heart Association Task Force on Practice Guidelines. J Am Coll Cardiol 2014;63(25 Pt B):2935-2959.

19. Wallis EJ, Ramsay LE, Jackson PR. Cardiovascular and coronary risk estimation in hypertension management. Heart 2002;88:306-312.

20. Taylor AJ. Atherosclerosis imaging to detect and monitor cardiovascular risk. Am J Cardiol 2002;90(10C):8L-11L.

21. Mattace-Raso FU, van der Cammen TJ, Hofman A, et al. Arterial stiffness and risk of coronary heart disease and stroke: the Rotterdam Study. Circulation 2006;113:657-663.

22. Vlachopoulos C, Aznaouridis K, Stefanadis C. Prediction of cardiovascular events and all-cause mortality with arterial stiffness: a systematic review and meta-analysis. J Am Coll Cardiol 2010;55:1318-1327.

23. Levey AS, Bosch JP, Lewis JB, Greene T, Rogers N, Roth D. A more accurate method to estimate glomerular filtration rate from serum creatinine: a new prediction equation. Modification of Diet in Renal Disease Study Group. Ann Intern Med 1999;130:461-470.

24. Song HG, Kim EJ, Seo HS, et al. Relative contributions of different cardiovascular risk factors to significant arterial stiffness. Int J Cardiol 2010;139:263-268.

25. Tsuchikura S, Shoji T, Kimoto E, et al. Brachial-ankle pulse wave velocity as an index of central arterial stiff- 
ness. J Atheroscler Thromb 2010;17:658-665.

26. Hofmann B, Riemer M, Erbs C, et al. Carotid to femoral pulse wave velocity reflects the extent of coronary artery disease. J Clin Hypertens (Greenwich) 2014;16:629-633.

27. Korjian S, Daaboul Y, El-Ghoul B, et al. Change in pulse wave velocity and short-term development of cardiovascular events in the hemodialysis population. J Clin Hypertens (Greenwich) 2016;18:857-863.

28. Ankle Brachial Index Collaboration, Fowkes FG, Murray GD, et al. Ankle brachial index combined with Framingham risk score to predict cardiovascular events and mortality: a meta-analysis. JAMA 2008;300:197-208.

29. Nambi V, Chambless L, Folsom AR, et al. Carotid intima-media thickness and presence or absence of plaque improves prediction of coronary heart disease risk: the ARIC (Atherosclerosis Risk In Communities) study. J Am Coll Cardiol 2010;55:1600-1607.

30. Higashi H, Okayama H, Saito M, et al. Relationship between augmentation index and left ventricular diastolic function in healthy women and men. Am J Hypertens 2013;26:1280-1286.

31. Holewijn S, den Heijer M, Kiemeney LA, Stalenhoef $\mathrm{AF}$, de Graaf J. Combining risk markers improves cardiovascular risk prediction in women. Clin Sci (Lond) 2014;126:139-146.

32. Verwoert GC, Elias-Smale SE, Rizopoulos D, et al. Does aortic stiffness improve the prediction of coronary heart disease in elderly? The Rotterdam Study. J Hum Hyper- tens 2012;26:28-34.

33. Ben-Shlomo Y, Spears M, Boustred C, et al. Aortic pulse wave velocity improves cardiovascular event prediction: an individual participant meta-analysis of prospective observational data from 17,635 subjects. J Am Coll Cardiol 2014;63:636-646.

34. Fujiwara Y, Chaves P, Takahashi R, et al. Relationships between brachial-ankle pulse wave velocity and conventional atherosclerotic risk factors in community-dwelling people. Prev Med 2004;39:1135-1142.

35. Jonason T, Henriksen E, Kangro T, Vessby B, Ringqvist I. Menopause is associated with the stiffness of the common carotid artery in 50-year-old women. Clin Physiol 1998;18:149-155.

36. Yamashina A, Tomiyama H, Takeda K, et al. Validity, reproducibility, and clinical significance of noninvasive brachial-ankle pulse wave velocity measurement. Hypertens Res 2002;25:359-364.

37. Kawai T, Ohishi M, Onishi M, et al. Prognostic impact of regional arterial stiffness in hypertensive patients. Heart Vessels 2015;30:338-346.

38. Munakata M. Brachial-ankle pulse wave velocity in the measurement of arterial stiffness: recent evidence and clinical applications. Curr Hypertens Rev 2014;10:49-57.

39. Michos ED, Nasir K, Braunstein JB, et al. Framingham risk equation underestimates subclinical atherosclerosis risk in asymptomatic women. Atherosclerosis 2006;184:201-206. 